\title{
Reliable 4G communication systems design using Idpc codes
}

\begin{abstract}
Generally, the multiple input multiple output (MIMO) transmission has been well known as one of the most important practical technique to combat channel fading as well as enhance the channel capacity of four generation $(4 \mathrm{G})$ mobile communication systems. There has been active research area on designing the efficient channel codes such as turbo codes and low density parity check (LDPC) codes for MIMO fading channels. Specially, LDPC codes have great attention due to their capacity approaching performance and flexibility in code design with low implementation complexity. Basically, the LDPC codes are a class of linear block codes with implementable decoders, which provide the near capacity performance on a large set of data transmission and data storage channels. In this paper, we investigate the performance of LDPC codes for MIMO 4G mobile communications systems. Simulation results show that 2 by 2 MIMO systems provide desired bit error performance in communication systems.
\end{abstract}

Keywords: channel code, LDPC, MIMO, parity check
Volume 5 Issue 2 - 2019

\author{
Md Masud Rana \\ Independent researcher, Australia
}

Correspondence: Md Masud Rana, Independent researcher, Sydney,Australia, Email mamaraece28@yahoo.com

Received: December 06, 2017 | Published: March 13, 2019

\section{Introduction}

There has been considerable activity toward the definition of next generation wireless local area networks (WLANs), which are expected to offer high-speed Internet services with data rates exceeding 100 $\mathrm{Mb} / \mathrm{s}$ and bring WLANs that are on par with wired Ethernet technology. However, the development of high data rate WLAN communication systems must cope with several implementation challenges, such as multipath fading as well as interference with limited transmission bandwidth and power. Now, it has been demonstrated that, for the predefined power and bandwidth, employing multiple transmit and receive antennas in an orthogonal frequency-division multiplexing (OFDM) system channels while minimizing equalization and symbol decoding complexity. ${ }^{1}$

Wireless technology has enormous potential to change the way people and things communicate. Wireless broadband access networks allow people and devices to have high speed connections to the backbone network from any place at any time. To minimize the bit error rate (BER), error correcting codes (ECCs) can play an important role for four generation $(4 \mathrm{G})$ mobile communication systems. The ECC is one of many tools made available for achieving consistent data transmission with lower BER. An ECC encoder generates redundant information according to the message information. The message and redundant information are transmitted when they may be corrupted. The decoder the exploits the redundancy in the received and garbled data and determines the original message information being send.

There are two types of ECCs such as a convolution codes and block code. Both types of codes have been widely used for error control in communication systems. Block codes can be divided into two categories, linear and nonlinear block codes. Nonlinear block codes are never used in practical applications and not much investigated. ${ }^{2} \mathrm{~A}$ code is said to be linear if any code words in the code can be added in modulo-2 arithmetic to produce a third code word in the code. The low density parity check codes (LDPCs) are linear block code that has been studied vastly in this decade. The main advantage of these codes is that they provide the performance at that very close to the capacity for a lot of different channels and linear time complex algorithms for decoding. ${ }^{1}$

LDPC codes were invented in 1962 by Robert Gallager. ${ }^{3-5}$ After the introduction of turbo codes, it was rediscovered by Mackay \& Neal, ${ }^{6,7}$ Wiberg $^{8}$ in the late 90 's. Gallager considered only regular LDPC codes. Regular codes are those which are represented by a sparse parity check matrix with a constant number of 'ones' in each column and in each row. In irregular LDPC codes, both weights become variable and performance of LDPC., ${ }^{9,10}$

For utilize of multiple antennas at the transmitter and/or receiver provides a much higher channel capacity compared to that of single antenna counterparts over wireless channels. With the current developments in iterative decoding, a number of pragmatic approaches using powerful turbo like codes such LDPC codes, repeat accumulate (RA) codes, or turbo codes themselves have been proposed to achieve a close capacity performance under a bit interleaved coded modulation (BICM) framework. For instance, by directly transmitting signals that are coded with an outer turbo code, it was shown in that a near capacity performance can be attained in a symmetric antenna setup where the number of receive antennas equals the number of transmit antennas. However, the error performance of such turbo coded systems experiences a severe degradation when the antenna scenario is asymmetric, i.e., the number of receive antennas is smaller than the number of transmit antennas. The LDPC coded modulation scheme that performs very close to the capacity limit, even when the antenna setup is asymmetric. ${ }^{11}$ Improvements made at the physical (PHY) layer of $4 \mathrm{~g}$ mobile communications systems, such as the use of multiple-input multiple-output (MIMO) antennas, are capable of greatly increasing their spectral efficiency.

In radio, multiple MIMO is the use of multiple antennas at both the transmitter and receiver to improve communication performance. It is one of several forms of smart antenna technology. MIMO technology has attracted attention in wireless communications, because it offers 
significant increases in data throughput and link range without additional bandwidth or transmit power. It achieves this by higher spectral efficiency and link reliability or diversity (reduced fading). Because of these properties, MIMO is an important part of modern wireless communication systems. In this paper, an overview LDPC code is presented for MIMO $4 \mathrm{G}$ mobile communication system. Simulation results show that 2 by 2 MIMO systems provide desired bit error performance in wireless communication systems.

The remainder of the paper is organized as follows: section II describes regular and irregular LDPC code. The communication system model is in section III. The simulation results are in section IV. The application of LDPC code in long term evolution (LTE) system in section V. Finally, some concluding remarks are given in section VI.

\section{Fundamental of LDPC code}

Generally speaking, the regular LDPC code is a linear block code whose parity check matrix $\mathrm{H}$ has column weight $\mathrm{g}$ and row weight $\mathrm{r}$, where $r=g(n / m)$ and $g<<m$. If $H$ is low density, but its row column weight are not both constant, then the code is an irregular LDPC code. For regular codes, the corresponding $\mathrm{H}$ matrix has $\beta_{\mathrm{r}}$ ones in each row and $\beta_{\mathrm{c}}$ one in each column. It means that every codeword bit participates in exactly $\beta_{c}$ parity-check equations and that every such check equation involves exactly $\beta_{\mathrm{r}}$ codeword bits. LDPC codes have been constructed mostly using regular random bipartite graphs. A graph whose nodes may be separated into two types, with edges connecting only nodes of different types is called bipartite graph. The two types of nodes in a bipartite graph are the variable nodes $(\mathrm{VN})$ and check nodes $(\mathrm{CN})$. The bipartite graph of a code is drawn as follows: $\mathrm{CN} i$ is connected to $\mathrm{VN} \mathrm{j}$ whenever element $h_{\mathrm{ij}}$ in $\mathrm{H}$ is a 1 . Observe from this rule that there are $\mathrm{m} \mathrm{CNs}$ in a bipartite graph, one for each check equation, and $\mathrm{n} \mathrm{VNs}$, one for each code bit. Further, the $\mathrm{m}$ rows of $\mathrm{H}$ specify the $\mathrm{m} \mathrm{CN}$ connections, and the $\mathrm{n}$ columns of $\mathrm{H}$ specify the $\mathrm{n}$ VN connections. ${ }^{2,17-19}$

Basically, the bipartite graph of a LDPC code acts as s blueprint for the iterative decoder in the following way. Each of the nodes acts as a locally operating processor and each edge acts as a bus that conveyed information from a given node to each of its neighbors. The information conveyed is typically probabilistic information, pertaining to the values of the bits assigned to the variable nodes. At the beginning of each half-iteration in the basic iterative decoding algorithm, each VN processor takes input from the channel and each of its neighboring CVs, and from these computes outputs for each one of its neighboring $\mathrm{CN}$ processors. In the next half- iteration, each $\mathrm{CN}$ processor takes input from each of its neighboring VNs, and from these computes outputs for each one of its neighboring VN processors. Here is an example of a regular parity check matrix with $\beta_{\mathrm{c}}=6$ and $\beta_{\mathrm{r}}=3$.

$$
\mathbf{H}=\left[\begin{array}{llllllllllll}
1 & 1 & 1 & 0 & 0 & 1 & 1 & 0 & 0 & 0 & 1 & 0 \\
1 & 1 & 1 & 1 & 1 & 0 & 0 & 0 & 0 & 0 & 0 & 1 \\
0 & 0 & 0 & 0 & 0 & 1 & 1 & 1 & 0 & 1 & 1 & 1 \\
1 & 0 & 0 & 1 & 0 & 0 & 0 & 1 & 1 & 1 & 0 & 1 \\
0 & 1 & 0 & 1 & 1 & 0 & 1 & 1 & 1 & 0 & 0 & 0 \\
0 & 0 & 1 & 0 & 1 & 1 & 0 & 0 & 1 & 1 & 1 & 0
\end{array}\right]
$$

The bipartite graph corresponding to this parity check matrix is shown in Figure 1. Here is an example of a regular parity check matrix with $\beta_{\mathrm{c}}=6$ and $\beta_{\mathrm{r}}=3$.

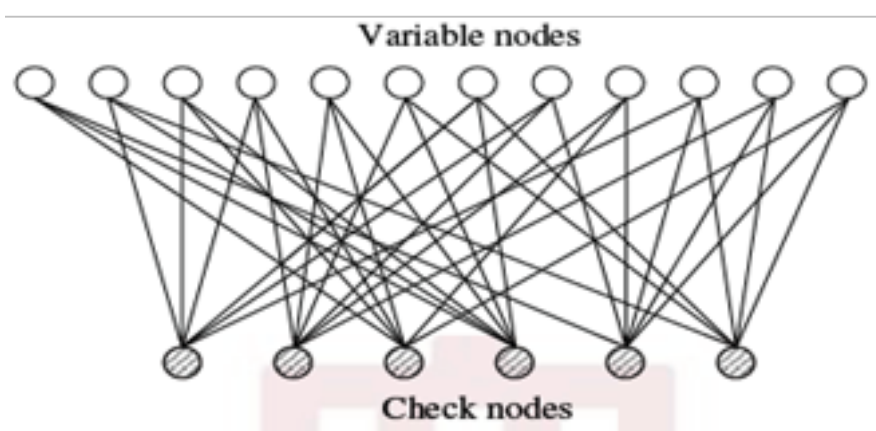

Figure I Bipartite graph corresponding to a regular parity check matrix. ${ }^{13}$

$$
\mathbf{H}=\left[\begin{array}{llllllllllll}
1 & 0 & 1 & 0 & 0 & 1 & 1 & 0 & 0 & 0 & 1 & 0 \\
1 & 1 & 0 & 1 & 1 & 0 & 0 & 0 & 0 & 0 & 0 & 1 \\
0 & 0 & 0 & 0 & 0 & 1 & 1 & 1 & 0 & 1 & 1 & 1 \\
1 & 0 & 0 & 1 & 0 & 0 & 0 & 1 & 1 & 1 & 0 & 1 \\
0 & 1 & 0 & 1 & 1 & 0 & 1 & 1 & 1 & 0 & 0 & 0 \\
0 & 0 & 1 & 0 & 1 & 1 & 0 & 0 & 1 & 1 & 1 & 0
\end{array}\right]
$$

The bipartite graph corresponding to this parity check matrix is shown in Figure 2.

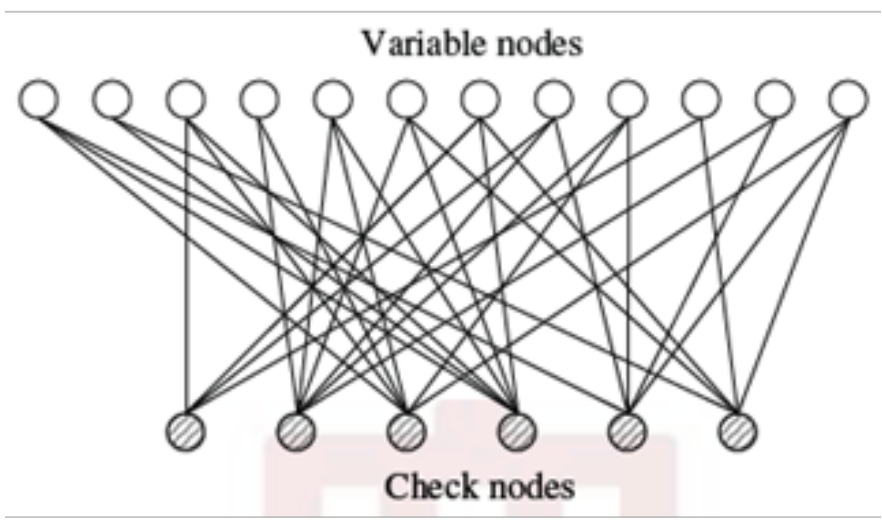

Figure 2 Bipartite graph corresponding to an irregular parity check matrix. ${ }^{13}$

\section{Design a reliable communication systems}

Generally, the digital communication systems are ubiquitous in our daily lives. The most obvious examples include cell phones, digital television via satellite or cable, digital radio, wireless internet connections via wireless fidelity (Wi-Fi) and worldwide interoperability for microwave access (WiMAX), and wired internet connection via cable modem. Additional examples include digital data storage devices, including magnetic disk drives, magnetic tape drives, optical disk drives, and flash drives. In the case of data-storage, information is communicated from one point in time to another rather than one point in space to another. A simplified block diagram for the communication system with channel coder is shown in Figure 3.

Actually, the information source may be originally in analog form and then later digitized, or it may be originally in digital form. The encoder is a processor that converts the information source bit sequence into an alternative bit sequence with a more efficient representation of the information, i.e., with fewer bits. Hence, this operation is often 
called compression. Depending on the source, the compression can be lossless or lossy. The role of the channel encoder is to protect the bits to be transmitted over a channel subject to noise, distortion, and interference. It does so by converting its input into an alternate sequence possessing redundancy, whose role is to provide immunity from the various channel impairments. The ratio of the number of bits that enter the channel encoder to the number that depart from it is called the code rate. The modulator converts the channel encoder output bit stream into a form that is appropriate for the channel. The channel is the physical medium through which the modulator output is conveyed. Physically, the channel can include antennas, amplifiers, and filters, both at the transmitter and at the receiver at the ends of the systems. $^{2}$

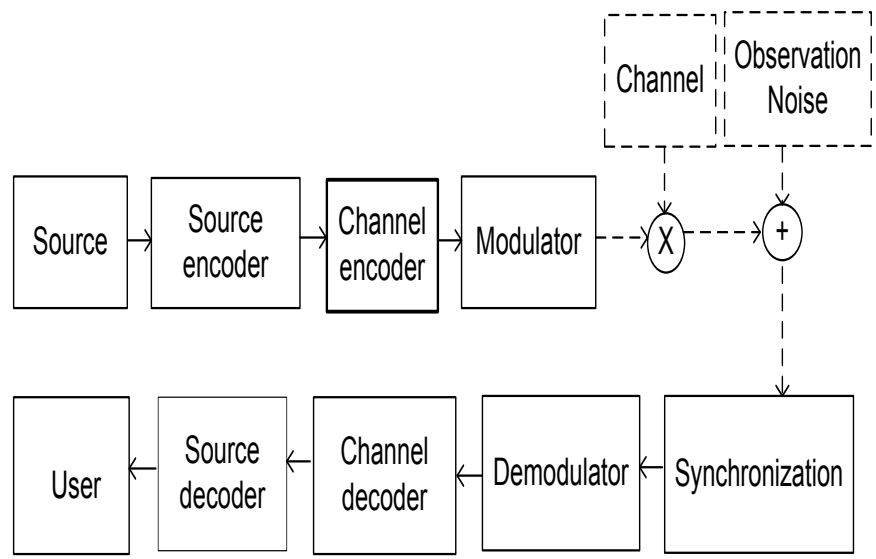

Figure 3 Basic block diagram of digital communication system.

Generally speaking, the demodulation is the modulator's counterpart which recovers the modulator input sequence from the modulator output sequence. The main function of the channel encoder in spite of the presence of noise, distortion, and interference in the received word. The source decoder is the encoder's counterpart which recovers the source sequence exactly, in the case of lossless compression, or approximately, in the case of lossy compression, from the encoder's output sequence. .,20-22 $^{2}$

In MIMO systems, multiple antennas are used at the transmitting end as well as at the receiving end. These systems have emerged as a most promising technology in the measures of higher data rates, better quality of service (QoS), and higher network capacity. Multiplicative increase in capacity, spectral efficiency, dramatic reductions of fading, increased system capacity (number of users), and improved resistance to interference (Figure 4).

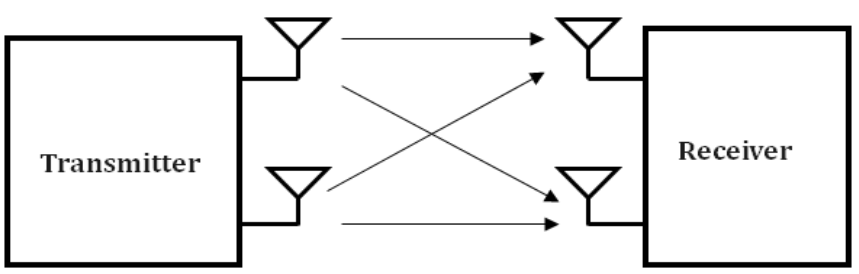

Figure 4 MIMO communication systems.

\section{Simulation results and discussion}

LDPCs are codes specified by a matrix containing mostly 0 's and relatively few 1's. The implementation of LDPC decoders tends to be complex and solutions for less complex decoders are a focal point of research. Stochastic computation was introduced in the 1960's as a method to design low precision digital circuits. This method has been used for iterative decoding of some specific error-correcting codes. The main advantage of this method is that probabilities can be manipulated using very simple circuits. ${ }^{13}$ The performance of the aforementioned is explored by performing extensive computer simulations. We used BPSK modulation, flat fading channel, and zero forcing (ZF) spatial decoding for our simulation. In addition in simulations, the receiver is assumed to know the channel exactly and employ an iterative detection and decoding algorithm. From the simulation result, it is apparent that 2 by 2 MIMO systems provide desired bit error performance in wireless communication systems (Figure 5).

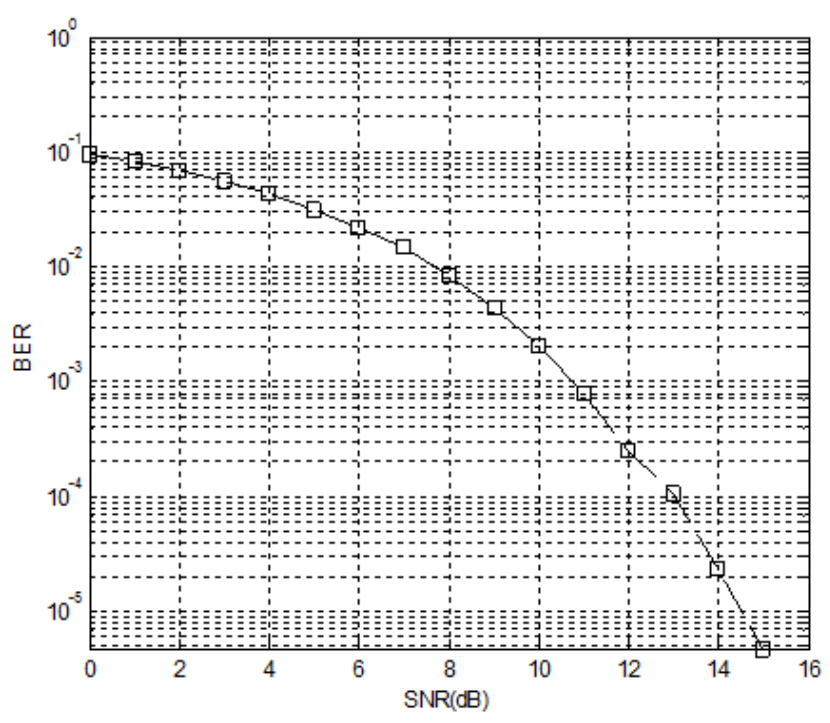

Figure $52 \times 2$ MIMO Gaussian channel with BPSK modulation.

\section{Application of LDPC code to LTE systems}

The further increasing demand on high data rates in wireless communication systems has arisen in order to support broadband services. The third generation partnership project (3GPP) LTE members started feasibility study on the enhancement of the UTRA in December 2004, to improve the mobile phone standard to cope with future requirements. The main purposes of the 3GPP LTE are substantially improved end-user throughputs, low latency, reduced user equipment (UE) complexity, high data rate, and significantly improved user experience with full mobility. First 3GPP LTE and LTE-advanced (LTE-A) specification is being finalized within 3GPP release 9 and release 10 , respectively. ${ }^{13-15}$

Specifically, the physical layer has become quite stable recently for a first implementation. LTE supports MIMO with one, two, four, and eight antenna elements at base station (BS) and mobile terminal. Both closed and open loop MIMO operation is possible. The target of 3GPP LTE-A is to reach and surpass the international telecommunication union (ITU) requirements. One of the important LTE-A benefits is the ability to leverage advanced topology networks; optimized heterogeneous networks with a mix of macros with low power nodes such as picocells, femtocells, ensures user fairness, worldwide roaming, and new relay nodes. ${ }^{14}$

LTE uses orthogonal frequency division multiple access (OFDMA) for downlink and single carrier-frequency division multiple access (SC-FDMA) for uplink transmission. ${ }^{1}$ A highly efficient way to 
cope with the frequency selectivity of wideband channel is OFDMA. OFDMA is an effective technique for combating multipath fading and for high bit rate transmission over mobile wireless channels. Turbo code is used in OFDMA systems as a tool for channel coding. But turbo coding suffers from the high decoding complexity as well as relatively high latency which make them unsuitable for some applications. LDPC can be a potential candidacy in this regard.

\section{Conclusion and future research}

Usually, the LDPC codes have great interest due to their capacity approaching performance and flexibility in code design with low implementation complexity. Basically, the LDPC codes are a class of linear block codes with implementable decoders, which provide near-capacity performance on a large set of data transmission and data-storage channels. In this paper, we investigate the application of LDPC codes to MIMO communication systems. Simulation results show that 2 by 2 MIMO systems provide desired bit error performance in wireless communication systems. In future, we will design a reliable communication system considering cyber attacks.

\section{Acknowledgments}

The author would like to thanks Yang Xiao.

\section{Conflicts of interest}

Author declares that there is none of the conflicts.

\section{References}

1. Xin Y, Mujtaba SA, Zhang T, et al. Bypass decoding: a reduced-complexity decoding technique for LDPC-coded MIMO-OFDM systems. IEEE Trans. On Vehicular Technology. 2008;57(4):2319-2333.

2. Ryan WE, Lin S. Channel codes classical and modern. Cambridge University Press. 2009.710 p.

3. Prasartkaew C, Choomchuay S. A parity check matrix design for irregular LDPC codes with $2 \mathrm{~K}$ block length. In Proc. International Symposium on Intelligent Signal Processing and Communication Systems. 2009. p. 7-9.

4. Gallager RG. Low density parity check codes. IRE transactions on Inform. Theory. $1962.8 \mathrm{p}$.

5. Gallager RG. Low density parity check codes. Cambridge: MIT Press; 1963.

6. Mackay DJC, Neal RM. Near Shannon limit performance of low density parity check codes. IEE Electron Letter. 1996;32(18):1645-1646.

7. Mackay DJC. Good error-correcting codes based on very sparse matrices. IEEE Trans Inform Theory. 1999;45(2):399-431.
8. Wiberg N. Codes and decoding on general graphs. Linkoeping studies in science and technology. 1996. 440 p.

9. Richardson TJ, Shokrollahi A, Urbanke R. Design of capacity approaching low-density parity-check codes. IEEE Trans. Inform. Theory. 2001;47(2):619-637.

10. Rakibul M, Kim J. On the use of QC-LDPC code for data transfer using short and medium block length. In Proc ICACT. 2009. p. 15-18.

11. Tran NH, Ngoc TL, Matsumoto T, et al. Achieving near-capacity performance on multiple-antenna channels with a simple concatenation scheme. IEEE Trans. On Wireless Comm. 2010;58(4):1048-1059.

12. Zhang J, Lee HN. Performance analysis on LDPC-coded systems over quasi-static (MIMO) fading channels. IEEE Trans. On Wireless Comm. 2000;56(12):2080-2093.

13. Rakibul M, Kim J. Capacity and BER analysis for Nakagami-n channel in LDPC coded wireless sensor network. In Proc. the International Conference on Intelligent Sensors, Sensor Networks and Information Processing (ISSNIP). 2008. p. 15-18.

14. Q Li, G Li, W Lee, et al. MIMO techniques in WiMAX and LTE: a feature overview. IEEE Commun. Magazine. 2010;48(5):86-92.

15. Noune M, Nix A. Frequency-domain precoding for single carrier frequency-division multiple access. IEEE Commun Magazine. 2010;48(5):86-92.

16. Yang Xiao. MIMO Multiple Antenna Wireless Communication Systems. Press of Posts and Telecommunications. 2009.

17. Rana MM, Rahaman MM, Kabir ME. Performance analysis of LDPC codes for MIMO 4G mobile communication systems. In Proc. of the International Conference on Electrical Computer and Telecommunication Engineering. 2012.

18. Lu Ben, Guosen Yue, Xiaodong Wang. Performance analysis and design optimization of LDPC-coded MIMO OFDM systems. IEEE Transactions on Signal Processing. 2004;52(2):348-361.

19. Lu Ben, Xiaodong Wang, Krishna R Narayanan. LDPC-based spacetime coded OFDM systems over correlated fading channels: Performance analysis and receiver design. IEEE Transactions on Communications. 2002;50(1):74-88.

20. Li Yan, William E Ryan. Bit-reliability mapping in LDPC-coded modulation systems. IEEE Communications Letters. 2005;9(1):1-3.

21. Djordjevic Ivan B, Bane Vasic, Mark Neifeld. LDPC-coded OFDM for optical communication systems with direct detection. IEEE journal of selected topics in quantum electronics. 2007;13(5):1446-1454.

22. Huang Jie, Shengli Zhou, Peter Willett. Nonbinary LDPC coding for multicarrier underwater acoustic communication. IEEE Journal on Selected Areas in Communication. 2008;26(9):1684-1696. 